\title{
The global trends and regional differences in incidence and mortality of hepatitis A from 1990 to 2019 and implications for its prevention
}

\author{
Guiying $\mathrm{Cao}^{1} \cdot$ Wenzhan Jing ${ }^{1} \cdot \mathrm{Jue} \mathrm{Liu}^{1} \cdot \mathrm{Min} \mathrm{Liu}^{1}{ }^{10}$
}

Received: 26 April 2021 / Accepted: 1 July 2021 / Published online: 3 August 2021

(c) The Author(s) 2021

\begin{abstract}
Background and purpose Despite decades of improved sanitation and hygiene measures and vaccine introduction, hepatitis A has been spread through numerous outbreaks globally. We used data from the Global Burden of Disease (GBD) study to quantify hepatitis A burden at the global, regional and national levels.

Methods Annual incident cases, deaths, age-standardized incidence rates (ASIRs), and age-standardized mortality rates (ASMRs) of hepatitis A between 1990 and 2019 were derived from the GBD study 2019. Percentage changes of cases and deaths, and estimated annual percentage changes (EAPCs) of ASIRs and ASMRs were calculated to quantify their temporal trends.

Results Global hepatitis A incident cases increased by $13.90 \%$ from 139.54 million in 1990 to 158.94 million in 2019. ASIR of hepatitis A remained stable (EAPC $=0.00,95 \% \mathrm{CI}-0.01$ to 0.01 ), whereas ASMR decreased (EAPC $=-4.63,95 \%$ CI -4.94 to -4.32 ) between 1990 and 2019. ASIR increased in low (EAPC $=0.09,95 \%$ CI 0.04 to 0.14 ) and low-middle $(\mathrm{EAPC}=0.04,95 \% \mathrm{CI} 0.03$ to 0.06$)$ socio-demographic index (SDI) regions. For GBD regions, the most significant increases of ASIR were detected in high-income Asia Pacific (EAPC $=0.53$, 95\% CI 0.41 to 0.66 ), Oceania (EAPC $=0.31,95 \%$ CI 0.25 to 0.36 ), and Australasia (EAPC $=0.28,95 \% \mathrm{CI} 0.13$ to 0.44 ). EAPC of ASIR was positively associated with SDI value in countries and territories with SDI value $\geq 0.7(\rho=-0.310, p<0.001)$.

Conclusion There is an unfavorable trend that hepatitis $\mathrm{A}$ is still pending in hyperendemic regions and is emerging in low endemic regions. These highlight the need of targeted and specific strategies to eliminate hepatitis A, such as sanitation measures and a comprehensive plan for surveillance and vaccination against hepatitis A.
\end{abstract}

Keywords Hepatitis A · Age-standardized incidence rate $\cdot$ Age-standardized mortality rate $\cdot$ Trend $\cdot$ Global burden of disease $\cdot$ Prevention

\section{Introduction}

Hepatitis A caused by hepatitis A virus (HAV) is the most common form of acute viral hepatitis globally [1]. HAV first identified by Feinstone and colleagues, is a member of

Guiying Cao and Wenzhan Jing have contributed equally and share the first authorship.

\footnotetext{
Guiying Cao

caoguiying@bjmu.edu.cn

Min Liu

liumin@bjmu.edu.cn

1 Department of Epidemiology and Biostatistics, School of Public Health, Peking University, Haidian District, No. 38 Xueyuan Road, Beijing 100191, China
}

the Hepatovirus genus of the family Picornaviridae [2, 3]. $\mathrm{HAV}$ is transmitted primarily through ingestion of food and water contaminated with the feces of an infected person or through close physical contact with an infectious person [4]. The World Health Organization (WHO) estimates more than 100 million HAV infections, causing approximately 1.5 million clinical cases of hepatitis A each year [5]. Symptomatic patients of hepatitis A can experience mild to severe illness, including nausea, vomiting, malaise, diarrhea, abdominal discomfort, fever, dark-colored urine, jaundice, and fulminant hepatitis, which is often fatal $[6,7]$. HAV infection is the second largest cause of fulminant viral hepatitis characterized by massive hepatocyte necrosis and inflammatory infiltrate after hepatitis B virus (HBV) infection [8]. In addition, superinfection of HAV with HBV is also a health 
problem in regions with high endemicity levels both of HAV and HBV [9].

After transmission of HAV, the virus enters the blood stream to gain access to the liver, the primary site of its replication, and induces liver injury. The pathogenesis of hepatitis $\mathrm{A}$ and the mechanisms underlying hepatocellular injury remain incompletely understood. Recent evidence suggests that gangliosides function as essential endosomal receptors for quasi-enveloped and naked HAV [10]. In addition, one previous study has reported that free fatty acids or high-concentration glucose enhances HAV replication in association with a reduction in glucose-regulated protein 78 expression [11]. Virus-specific cytotoxic T lymphocytes play a pivotal role in clearing virus-infected cells but also a major driver for HAV-associated immunopathology that may also cause liver injury [11]. Natural killer (NK) cells, NKT cells, and non-HAV-specific $\mathrm{CD}^{+} \mathrm{T}$ cells have been reported to contribute to liver injury [11-13]. In addition, intrinsic apoptosis of virus-infected hepatocytes has been implicated as a cause of liver injury in a murine model of HAV infection [14]. Finally, the severity of HAV-induced liver injury may be linked to genetic variations in both host and viral factors $[11,15]$. Since hepatitis A is clinically indistinguishable from diseases caused by other hepatitis viruses, specific serum markers and nucleic acid detection techniques are required to make the diagnosis [16]. Immunoglobulin M anti-HAV increases in the acute setting and confirms recent exposure to HAV, whereas immunoglobulin $\mathrm{G}$ anti-HAV is elevated during convalescence and indicates past exposure. HAV nucleic acid sequencing is performed on polymerase chain reaction products to provide the ultimate means of identifying and characterizing the organism.

Hepatitis A vaccine is highly immunogenic and well tolerated in preventing hepatitis A [17]. Several randomized controlled trials reported that the efficacy of hepatitis A vaccine used for pre-exposure prophylaxis and post-exposure prophylaxis ranged from 95 to $100 \%$ among children [18-21]. However, hepatitis A vaccine was used or planned to introduce in routine immunization of children in only 34 countries as of May 2019 worldwide. In addition to the introduction of hepatitis A vaccine, decades of improved sanitation and hygiene measures contribute to combat hepatitis A. The sanitary conditions and hygienic practices were good in high-income countries but still poor in low- and middle-income countries. However, hepatitis A outbreaks frequently occurred in countries regardless of their income in recent years [22-24]. In high-income countries, imported frozen produce items and infected travelers from endemic regions are associated with hepatitis A outbreaks [24-26]. In addition, hepatitis A outbreaks frequently occurred among the men who have sex with men (MSM) and persons who injected drugs (PWIDs) in high-income countries [27, 28]. Frequent international trades and travels in middle-income countries play important roles in outbreaks of hepatitis A and global transmissions of HAV [24]. For low-income countries, the endemicity levels of hepatitis A are still high due to poor sanitary conditions and hygienic practices [24]. The prevention of hepatitis A is facing more and more complex situations, especially with globalization processes. Therefore, understanding the endemicity levels of hepatitis A at the global, regional and national levels and targeted strategies for its prevention were essential for achieving the goal of eliminating viral hepatitis by 2030 .

In this current study, we extracted detailed data of the incidence and mortality of hepatitis A from the Global Burden of Disease (GBD) Study 2019 to assess the temporal trends in incidence and mortality of hepatitis A at global, regional, and national levels from 1990 to 2019. Our study can extend and complement the previous study [29], while also providing a more comprehensive perspective in the design of targeted strategies in hepatitis A prevention tailored to different countries.

\section{Methods}

\section{Data source}

This study used data of annual incident cases, deaths, agestandardized incidence rates (ASIRs), and age-standardized mortality rates (ASMRs) of hepatitis A from 1990 to 2019, by sex, age, and location, collected from the Global Health Data Exchange (GHDx) query tool (http://ghdx.healthdata. org/gbd-results-tool) [30]. Data were available from a total of 204 countries and territories, and these were categorized into 5 regions in terms of socio-demographic index (SDI) and 21 GBD regions according to geographical contiguity. The 21 GBD regions, including 5 low-income regions, 12 middle-income regions, and 4 high-income regions, are listed in Table S1. Specific methods of GBD study 2019 estimation process for the incidence of hepatitis A were described elsewhere [31]. Briefly, age-specific anti-HAV immunoglobulin $\mathrm{G}$ seroprevalence data from populationbased studies and surveys were reviewed to estimate the incidence of hepatitis A using the Bayesian meta-regression tool by DisMod-MR 2.1 model [31]. The number of populations in 204 countries and territories of GBD study was collected from the United Nations, Department of Economic and Social Affairs, Population Division (2019) (https://popul ation.un.org/wpp/) [24]. 


\section{SDI}

The SDI is a composite indicator of development status strongly correlated with health outcomes [30]. It is the geometric mean of 0 to 1 indices of lag distributed income per capita, average years of schooling for those ages 15 and older, and total fertility rate under the age of 25 . A location with an SDI of 0 indicates a theoretical minimum level of development status relevant to health outcomes, while a location with an SDI of 1 indicates a theoretical maximum level [30]. According to SDI values in 2019, the 204 countries and territories were divided into five regions, including low, low-middle, middle, high-middle, and high SDI regions. The SDI values of 204 countries and territories in 2019 were shown in Table S2.

\section{Statistical analysis}

The percentage changes in hepatitis A incident cases and deaths, and the estimated annual percentage changes (EAPCs) of ASIRs and ASMRs were calculated to quantify the trends in incidence and mortality of hepatitis A. To compare the incidence and mortality rates of hepatitis A across different populations, the ASIRs and ASMRs were carried out by applying the age-specific rates for each location, sex and year to a GBD World Standard Population to adjust for potential confounding of age structure [32]. The percentage changes in hepatitis A incident cases from

1990 to 2019 were calculated by the equation: Percentage change $=\frac{\text { Incident cases in } 2019-\text { Incident cases in } 1990}{\text { Incident cases in } 1990} \times 100 \%$. Similar with the calculation of percentage changes in incident cases, the percentage changes in hepatitis A death and number of populations were calculated. EAPC is a summary and widely used measure of the ASIR and ASMR tend over a specified time interval. A regression line was fitted to the natural logarithm of the ASIR, i.e., $y=\alpha+\beta x+\varepsilon$, where $y=\ln$ (ASIR) and $x=$ calendar year. EAPC was calculated as $100 \times\left(e^{\beta}-1\right)$ and its $95 \%$ confidence interval (CI) was calculated to reflect the temporal trend in ASIR. Similar with the calculation of EAPC of ASIR, the EAPC of ASMR was calculated. The trends in ASIR and ASMR were reflected in the EAPC value and its 95\% CI: ASIR and ASMR are in an upward trend when the EAPC and the lower boundary of the $95 \% \mathrm{CI}$ are positive; conversely, ASIR and ASMR are in a downward trend when EAPC and the upper boundary of the $95 \% \mathrm{CI}$ are negative.

Moreover, the correlations between EAPC and ASIR (1990) as well as SDI values (2019) in different countries and territories were evaluated by Pearson correlation analyses to define the potential factors affecting EAPC.
The polynomial curves were also modeled. All analyses were conducted with SAS 9.4 (SAS Institute, Inc., Cary, NC) and Origin 2019b. A two-tailed $p$ value less than 0.05 was considered statistically significant.

\section{Results}

\section{Global trends in incidence and mortality rates of hepatitis A}

Globally, the number of hepatitis A incident cases increased by $13.90 \%$ from 139.54 million in 1990 to 158.94 million in 2019 , whereas the number of hepatitis A deaths decreased by $63.61 \%$ from 10.79 in 1990 thousand to 3.93 thousand in 2019 (Table 1). The overall ASIR of hepatitis A remained stable $(\mathrm{EAPC}=0.00,95 \% \mathrm{CI}-0.01$ to 0.01 ) from 2263.97 per 100,000 in 1990 to 2272.08 per 100,000 in 2019, whereas the overall ASMR of hepatitis A decreased $(\mathrm{EAPC}=-4.63,95 \% \mathrm{CI}-4.94$ to -4.32$)$ from 2.07 per 100,000 in 1990 to 0.52 per 100,000 in 2019 (Table 2).

The absolute number of hepatitis A incident cases in India (30.39 million) and China (19.37 million) approximately accounted for one-third of hepatitis A incident cases of the global (158.94 million) in 2019 (Table S2). The countries with the most pronounced increase of hepatitis A incident cases were Qatar (323.67\%) and Afghanistan (246.45\%) (Table S2 and Fig. 1A). The ASIR of hepatitis A varies considerably across the world, with the highest ASIR in Comoros (2686.34 per 100,000 in 2019), followed by Djibouti and Tanzania (Fig. 1B). The ASIRs were deemed in an increasing trend in 65 countries or territories, with the largest increase in Thailand (EAPC $=1.35 ; 95 \%$ CI 0.99 to 1.71 ), followed by Japan and Germany (Fig. 1C and Table S2). The ASRs remained stable in 35 countries or territories, including the United States of America (USA), Mexico, and Singapore. The remaining 65 countries or territories showed a decreasing trend in ASRs, with the highest decrease in Belarus (EAPC $=-0.76 ; 95 \% \mathrm{CI}-0.89$ to -0.64 ), followed by Argentina and Romania (Fig. 1C and Table S2).

\section{Trends in incidence and mortality rates of hepatitis $A$ across five SDI regions}

For the five SDI regions, the number of hepatitis A incident cases increased in low $(74.90 \%)$, low-middle $(11.80 \%)$, and high $(6.67 \%)$ SDI regions but decreased in middle (7.74\%) and middle-high (14.81\%) SDI regions (Table 1). The number of hepatitis A deaths decreased in all five SDI regions, with the largest decreased in middlehigh SDI region (84.84\%) (Table 1). The ASIR increased in low (EAPC $=0.09 ; 95 \%$ CI 0.04 to 0.14$)$ and low-middle $(\mathrm{EAPC}=0.04 ; 95 \%$ CI 0.03 to 0.06$)$ SDI regions but 
Table 1 The incident cases and deaths of hepatitis A and population in 1990 and 2019 and their percentage change from 1990 to 2019

\begin{tabular}{|c|c|c|c|c|c|c|c|c|c|}
\hline \multirow{2}{*}{$\begin{array}{l}\text { Character- } \\
\text { istics }\end{array}$} & \multicolumn{3}{|c|}{ Incident cases } & \multicolumn{3}{|l|}{ Deaths } & \multicolumn{3}{|l|}{ Population } \\
\hline & $\begin{array}{l}1990 \\
\text { No. } \times 10^{5} \\
(95 \% \text { UI })\end{array}$ & $\begin{array}{l}2019 \\
\text { No. } \times 10^{5} \\
(95 \% \text { UI })\end{array}$ & $\begin{array}{l}\text { Percentage } \\
\text { change } \\
(\%)\end{array}$ & $\begin{array}{l}1990 \\
\text { No. } \times 10^{3} \\
(95 \% \text { UI })\end{array}$ & $\begin{array}{l}2019 \\
\text { No. } \times 10^{3} \\
(95 \% \text { UI })\end{array}$ & $\begin{array}{l}\text { Percentage } \\
\text { change } \\
(\%)\end{array}$ & $\begin{array}{l}1990 \\
\text { No. } \times 10^{5}\end{array}$ & $\begin{array}{l}2019 \\
\text { No. } \times 10^{5}\end{array}$ & $\begin{array}{l}\text { Percentage } \\
\text { change } \\
(\%)\end{array}$ \\
\hline Overall & $\begin{array}{c}1395.44 \\
(1303.39- \\
1484.48)\end{array}$ & $\begin{array}{c}1589.44 \\
(1492.31- \\
1690.85)\end{array}$ & 13.90 & $\begin{array}{r}107.92 \\
(82.49- \\
133.25)\end{array}$ & $\begin{array}{l}39.28 \\
(27.99- \\
52.35)\end{array}$ & -63.61 & 53182.09 & 77010.07 & 44.80 \\
\hline \multicolumn{10}{|l|}{ SDI } \\
\hline Low & $\begin{array}{l}223.05 \\
\quad(208.56- \\
237.30)\end{array}$ & $\begin{array}{c}390.12 \\
\quad(364.06- \\
415.51)\end{array}$ & 74.90 & $\begin{array}{l}22.58 \\
(14.57- \\
31.50)\end{array}$ & $\begin{array}{c}13.16(9.03- \\
17.94)\end{array}$ & -41.73 & 4272.77 & 9416.38 & 120.38 \\
\hline Low-middle & $\begin{array}{l}377.05 \\
(352.73- \\
402.77)\end{array}$ & $\begin{array}{l}421.56 \\
(395.19- \\
449.50)\end{array}$ & 11.80 & $\begin{array}{l}52.20 \\
\quad(39.62- \\
64.40)\end{array}$ & $\begin{array}{c}19.79 \\
(13.43- \\
27.76)\end{array}$ & -62.08 & 13911.82 & 22561.46 & 62.17 \\
\hline Middle & $\begin{array}{c}469.65 \\
(436.39- \\
502.51)\end{array}$ & $\begin{array}{c}434.57 \\
\quad(404.34- \\
464.46)\end{array}$ & -7.47 & $\begin{array}{l}27.50 \\
\quad(20.60- \\
34.37)\end{array}$ & $\begin{array}{l}5.40(3.07- \\
8.78)\end{array}$ & -80.37 & 21411.40 & 28885.04 & 34.90 \\
\hline Middle-high & $\begin{array}{l}235.45 \\
\quad(217.05- \\
253.19)\end{array}$ & $\begin{array}{c}200.58 \\
(185.08- \\
215.46)\end{array}$ & -14.81 & $\begin{array}{l}5.29(3.94- \\
6.62)\end{array}$ & $\begin{array}{l}0.80(0.47- \\
1.47)\end{array}$ & -84.84 & 6120.66 & 7067.99 & 15.48 \\
\hline High & $\begin{array}{l}89.47 \\
(81.33- \\
97.53)\end{array}$ & $\begin{array}{l}95.44 \\
(87.17- \\
103.48)\end{array}$ & 6.67 & $\begin{array}{l}0.33(0.26- \\
0.44)\end{array}$ & $\begin{array}{l}0.11(0.08- \\
0.15)\end{array}$ & -65.65 & 7465.43 & 9079.20 & 21.62 \\
\hline \multicolumn{10}{|l|}{ GBD region } \\
\hline $\begin{array}{l}\text { High-income } \\
\text { Asia Pacific }\end{array}$ & $\begin{array}{l}17.76 \\
(16.09- \\
19.35)\end{array}$ & $\begin{array}{c}15.63 \\
(14.23- \\
17.09)\end{array}$ & -12.03 & $\begin{array}{l}0.05(0.04- \\
0.06)\end{array}$ & $\begin{array}{l}0.01(0.01- \\
0.02)\end{array}$ & -68.57 & 1706.95 & 1843.23 & 7.98 \\
\hline Central Asia & $\begin{array}{l}22.42 \\
(20.65- \\
24.08)\end{array}$ & $\begin{array}{l}23.51 \\
\quad(21.70- \\
25.27)\end{array}$ & 4.87 & $\begin{array}{l}0.82(0.61- \\
1.04)\end{array}$ & $\begin{array}{l}0.08(0.05- \\
0.10)\end{array}$ & -90.54 & 684.98 & 934.39 & 36.41 \\
\hline East Asia & $\begin{array}{l}287.42 \\
\quad(265.07- \\
309.31)\end{array}$ & $\begin{array}{c}200.07 \\
(182.44- \\
216.67)\end{array}$ & -30.39 & $\begin{array}{c}12.90(7.97- \\
17.54)\end{array}$ & $\begin{array}{l}0.63(0.43- \\
0.87)\end{array}$ & -95.12 & 12176.55 & 14832.24 & 21.81 \\
\hline South Asia & $\begin{array}{c}360.26 \\
(335.30- \\
388.00)\end{array}$ & $\begin{array}{l}420.60 \\
(388.3- \\
453.03)\end{array}$ & 16.75 & $\begin{array}{c}67.09 \\
(50.34- \\
81.66)\end{array}$ & $\begin{array}{c}27.27 \\
(17.74- \\
39.71)\end{array}$ & -59.36 & 11035.34 & 17754.01 & 60.88 \\
\hline $\begin{array}{l}\text { Southeast } \\
\text { Asia }\end{array}$ & $\begin{array}{c}120.29 \\
(109.37- \\
130.96)\end{array}$ & $\begin{array}{c}121.81 \\
(111.18- \\
132.18)\end{array}$ & 1.26 & $\begin{array}{l}8.64(4.74- \\
16.21)\end{array}$ & $\begin{array}{l}2.29(0.87- \\
4.21)\end{array}$ & -73.53 & 4598.68 & 6789.96 & 47.65 \\
\hline Australasia & $\begin{array}{l}2.02(1.80- \\
2.26)\end{array}$ & $\begin{array}{l}2.81(2.49- \\
3.10)\end{array}$ & 38.64 & $\begin{array}{l}0.00(0.00- \\
0.00)\end{array}$ & $\begin{array}{l}0.00(0.00- \\
0.00)\end{array}$ & 64.55 & 203.59 & 299.86 & 47.29 \\
\hline Caribbean & $\begin{array}{l}9.78(9.01- \\
10.55)\end{array}$ & $\begin{array}{l}10.16(9.33- \\
10.94)\end{array}$ & 3.91 & $\begin{array}{l}0.17(0.10- \\
0.24)\end{array}$ & $\begin{array}{l}0.06(0.03- \\
0.09)\end{array}$ & -63.85 & 342.60 & 437.63 & 27.74 \\
\hline $\begin{array}{l}\text { Central } \\
\text { Europe }\end{array}$ & $\begin{array}{c}19.80 \\
(17.82- \\
21.57)\end{array}$ & $\begin{array}{c}12.93 \\
(11.75- \\
14.06)\end{array}$ & -34.70 & $\begin{array}{l}0.09(0.05- \\
0.12)\end{array}$ & $\begin{array}{l}0.00(0.00- \\
0.01)\end{array}$ & -95.50 & 1229.58 & 1139.58 & -7.32 \\
\hline $\begin{array}{l}\text { Eastern } \\
\text { Europe }\end{array}$ & $\begin{array}{l}37.79 \\
\quad(34.26- \\
41.00)\end{array}$ & $\begin{array}{l}29.15 \\
(26.54- \\
31.49)\end{array}$ & -22.88 & $\begin{array}{l}0.04(0.03- \\
0.08)\end{array}$ & $\begin{array}{l}0.01(0.00- \\
0.01)\end{array}$ & -77.66 & 2214.37 & 2093.54 & -5.46 \\
\hline $\begin{array}{l}\text { Western } \\
\text { Europe }\end{array}$ & $\begin{array}{l}35.82 \\
(32.97- \\
38.77)\end{array}$ & $\begin{array}{l}37.00 \\
\quad(33.75- \\
40.31)\end{array}$ & 3.28 & $\begin{array}{l}0.11(0.08- \\
0.17)\end{array}$ & $\begin{array}{l}0.07(0.04- \\
0.09)\end{array}$ & -35.12 & 3813.61 & 4332.11 & 13.60 \\
\hline $\begin{array}{l}\text { Andean Latin } \\
\text { America }\end{array}$ & $\begin{array}{l}12.61 \\
(11.71- \\
13.54)\end{array}$ & $\begin{array}{l}15.76 \\
(14.61- \\
16.94)\end{array}$ & 24.97 & $\begin{array}{l}0.17(0.12- \\
0.22)\end{array}$ & $\begin{array}{l}0.03(0.02- \\
0.04)\end{array}$ & -81.29 & 391.67 & 613.97 & 56.76 \\
\hline
\end{tabular}


Table 1 (continued)

\begin{tabular}{|c|c|c|c|c|c|c|c|c|c|}
\hline \multirow{2}{*}{$\begin{array}{l}\text { Character- } \\
\text { istics }\end{array}$} & \multicolumn{3}{|c|}{ Incident cases } & \multicolumn{3}{|l|}{ Deaths } & \multicolumn{3}{|l|}{ Population } \\
\hline & $\begin{array}{l}1990 \\
\text { No. } \times 10^{5} \\
(95 \% \text { UI })\end{array}$ & $\begin{array}{l}2019 \\
\text { No. } \times 10^{5} \\
(95 \% \text { UI })\end{array}$ & $\begin{array}{l}\text { Percentage } \\
\text { change } \\
(\%)\end{array}$ & $\begin{array}{l}1990 \\
\text { No. } \times 10^{3} \\
(95 \% \text { UI })\end{array}$ & $\begin{array}{l}2019 \\
\text { No. } \times 10^{3} \\
(95 \% \text { UI })\end{array}$ & $\begin{array}{l}\text { Percentage } \\
\text { change } \\
(\%)\end{array}$ & $\begin{array}{l}1990 \\
\text { No. } \times 10^{5}\end{array}$ & $\begin{array}{l}2019 \\
\text { No. } \times 10^{5}\end{array}$ & $\begin{array}{l}\text { Percentage } \\
\text { change } \\
(\%)\end{array}$ \\
\hline $\begin{array}{l}\text { Central Latin } \\
\text { America }\end{array}$ & $\begin{array}{l}55.94 \\
\quad(52.08- \\
59.83)\end{array}$ & $\begin{array}{l}57.35 \\
\quad(53.68- \\
61.06)\end{array}$ & 2.52 & $\begin{array}{l}0.31(0.27- \\
0.37)\end{array}$ & $\begin{array}{l}0.15(0.12- \\
0.19)\end{array}$ & -52.60 & 1659.31 & 2560.51 & 54.31 \\
\hline $\begin{array}{l}\text { Southern } \\
\text { Latin } \\
\text { America }\end{array}$ & $\begin{array}{l}12.52 \\
(11.27- \\
13.61)\end{array}$ & $\begin{array}{l}11.84 \\
(10.63- \\
12.99)\end{array}$ & -5.44 & $\begin{array}{l}0.09(0.07- \\
0.10)\end{array}$ & $\begin{array}{l}0.01(0.01- \\
0.02)\end{array}$ & -84.42 & 490.03 & 671.94 & 37.12 \\
\hline $\begin{array}{l}\text { Tropical } \\
\text { Latin } \\
\text { America }\end{array}$ & $\begin{array}{l}39.36 \\
(36.37- \\
42.56)\end{array}$ & $\begin{array}{l}38.30 \\
\quad(34.98- \\
41.31)\end{array}$ & -2.68 & $\begin{array}{l}0.21(0.17- \\
0.30)\end{array}$ & $\begin{array}{c}0.06(0.04- \\
0.09)\end{array}$ & -72.55 & 1532.27 & 2180.94 & 42.33 \\
\hline $\begin{array}{l}\text { North Africa } \\
\text { and Middle } \\
\text { East }\end{array}$ & $\begin{array}{l}117.34 \\
(108.34- \\
126.35)\end{array}$ & $\begin{array}{l}145.52 \\
(134.95- \\
155.99)\end{array}$ & 24.02 & $\begin{array}{l}7.57(2.59- \\
12.69)\end{array}$ & $\begin{array}{l}2.18(0.78- \\
4.37)\end{array}$ & -71.21 & 3352.15 & 6107.59 & 82.20 \\
\hline $\begin{array}{l}\text { High-income } \\
\text { North } \\
\text { America }\end{array}$ & $\begin{array}{l}28.91 \\
\quad(25.85- \\
31.96)\end{array}$ & $\begin{array}{l}34.87 \\
(31.43- \\
38.22)\end{array}$ & 20.63 & $\begin{array}{l}0.09(0.08- \\
0.13)\end{array}$ & $\begin{array}{l}0.05(0.04- \\
0.07)\end{array}$ & -42.92 & 2797.17 & 3665.33 & 31.04 \\
\hline Oceania & $\begin{array}{l}1.88(1.69- \\
2.08)\end{array}$ & $\begin{array}{l}3.91(3.51- \\
4.29)\end{array}$ & 107.85 & $\begin{array}{l}0.08(0.02- \\
0.15)\end{array}$ & $\begin{array}{l}0.06(0.03- \\
0.12)\end{array}$ & -24.72 & 65.56 & 115.68 & 76.46 \\
\hline $\begin{array}{l}\text { Central Sub- } \\
\text { Saharan } \\
\text { Africa }\end{array}$ & $\begin{array}{l}24.68 \\
\quad(22.71- \\
26.55)\end{array}$ & $\begin{array}{l}49.09 \\
(45.35- \\
52.63)\end{array}$ & 98.94 & $\begin{array}{l}1.36(0.10- \\
3.26)\end{array}$ & $\begin{array}{l}0.71(0.15- \\
1.47)\end{array}$ & -48.05 & 529.93 & 1322.70 & 149.60 \\
\hline $\begin{array}{l}\text { Eastern Sub- } \\
\text { Saharan } \\
\text { Africa }\end{array}$ & $\begin{array}{l}87.90 \\
(82.02- \\
93.58)\end{array}$ & $\begin{array}{c}163.24 \\
(151.74- \\
173.83)\end{array}$ & 85.71 & $\begin{array}{l}4.45(2.42- \\
6.29)\end{array}$ & $\begin{array}{l}3.96(2.06- \\
6.79)\end{array}$ & -10.92 & 1849.04 & 4167.37 & 125.38 \\
\hline $\begin{array}{l}\text { Southern } \\
\text { Sub-Saha- } \\
\text { ran Africa }\end{array}$ & $\begin{array}{l}17.91 \\
(16.61- \\
19.16)\end{array}$ & $\begin{array}{l}20.74 \\
\quad(19.12- \\
22.26)\end{array}$ & 15.85 & $\begin{array}{l}0.19(0.05- \\
0.40)\end{array}$ & $\begin{array}{l}0.08(0.05- \\
0.13)\end{array}$ & -56.03 & 524.79 & 812.75 & 54.87 \\
\hline $\begin{array}{l}\text { Western Sub- } \\
\text { Saharan } \\
\text { Africa }\end{array}$ & $\begin{array}{c}83.03 \\
(77.09- \\
88.91)\end{array}$ & $\begin{array}{l}175.15 \\
(162.47- \\
187.36)\end{array}$ & 110.95 & $\begin{array}{l}3.49(1.19- \\
7.16)\end{array}$ & $\begin{array}{l}1.56(0.88- \\
2.98)\end{array}$ & -55.38 & 1983.91 & 4334.72 & 118.49 \\
\hline
\end{tabular}

GBD Global Burden of Disease, SDI Socio-Demographic Index, UI uncertainty interval

decreased in middle (EAPC $=-0.10 ; 95 \% \mathrm{CI}-0.15$ to $-0.05)$ and middle-high (EAPC $=-0.35 ; 95 \% \mathrm{CI}:-0.38$ to -0.31 ) SDI region (Table 2). The ASIR remained stable in high SDI regions (EAPC $=0.02 ; 95 \% \mathrm{CI}-0.03$ to 0.06 ). The ASMR decreased in all 5 SDI regions, with the largest decrease in middle-high SDI region $(\mathrm{EAPC}=-7.56$; $95 \%$ CI -7.76 to -7.37 ) (Fig. 2).

Figure 3 shows the number of incidents cases of hepatitis A by age at 5 SDI regions from 1990 to 2019. In low SDI regions, the growing number of incident cases of hepatitis $\mathrm{A}$ was due to the gradual increase in the number of cases in all age groups, especially in children aged under 5 years (Fig. 3A). The increased incidents cases of children aged under 5 years accounted for $50.16 \%$ of the total increased incident cases from 1990 to 2019. However, the number of incident cases was deceasing only in children aged under 5 years but increasing in other age groups in low-middle SDI regions. In middle and above SDI regions (including middle, middle-high, and high SDI regions), the number of incident cases was decreasing in children aged less than 15 years but increasing in population aged 15 years plus.

\section{Trends in incidence and mortality rates of hepatitis $A$ across $21 \mathrm{GBD}$ regions}

For the 21 GBD regions, the incident cases of hepatitis A increased even more in GBD regions with larger population increases, such as Western Sub-Saharan Africa (110.95\%), Oceania (107.85\%), Central SubSaharan Africa (98.94\%), and Eastern Sub-Saharan Africa $(85.71 \%$ ) (Table 1). Low-income regions suffered the severest threat of hepatitis A, with the highest ASIR of 2625.34 per 100,000 in Eastern Sub-Saharan Africa, followed by Western Sub-Saharan Africa (2625.34 per 100,000 in 2019) and Central Sub-Saharan Africa (2543.07 per 100,000 in 2019) in 2019 (Table 2). The trends in ASIRs of hepatitis A were heterogeneous 
Table 2 The ASIRs and ASMRs of hepatitis A in 1990 and 2019 and their temporal trends from 1990 to 2019

\begin{tabular}{|c|c|c|c|c|c|c|}
\hline \multirow[t]{2}{*}{ Characteristics } & \multicolumn{3}{|l|}{ ASIR per 100,000} & \multicolumn{3}{|c|}{ ASMR per 100,000} \\
\hline & $\begin{array}{l}1990 \\
\text { No. }(95 \% \text { UI })\end{array}$ & $\begin{array}{l}2019 \\
\text { No. }(95 \% \text { UI })\end{array}$ & $\begin{array}{l}\text { EAPC } \\
\text { No. }(95 \% \mathrm{CI})\end{array}$ & $\begin{array}{l}1990 \\
\text { No. }(95 \% \text { UI })\end{array}$ & $\begin{array}{l}2019 \\
\text { No. }(95 \% \text { UI })\end{array}$ & $\begin{array}{l}\text { EAPC } \\
\text { No. }(95 \% \text { CI })\end{array}$ \\
\hline Overall & $\begin{array}{l}2263.97(2120.55 \\
2404.67)\end{array}$ & $\begin{array}{l}2272.08(2121.79, \\
2421.80)\end{array}$ & $0.00(-0.01,0.01)$ & $2.07(1.55,2.56)$ & $0.52(0.37,0.69)$ & $-4.63(-4.94,-4.32)$ \\
\hline \multicolumn{7}{|l|}{ SDI } \\
\hline Low & $\begin{array}{l}2435.07(2274.58 \\
2597.09)\end{array}$ & $\begin{array}{l}2379.07(2215.87, \\
2535.86)\end{array}$ & $0.09(0.04,0.14)$ & $5.31(3.09,7.71)$ & $1.62(1.07,2.19)$ & $-4.32(-4.74,-3.91)$ \\
\hline Low-middle & $\begin{array}{l}2326.27(2178.11 \\
2490.21)\end{array}$ & $\begin{array}{l}2339.91(2185.20, \\
2499.61)\end{array}$ & $0.04(0.03,0.06)$ & $5.10(3.86,6.42)$ & $1.25(0.86,1.72)$ & $-4.79(-5.04,-4.54)$ \\
\hline Middle & $\begin{array}{l}2302.94(2143.00 \\
2461.55)\end{array}$ & $\begin{array}{l}2137.82(1972.97, \\
2300.65)\end{array}$ & $\begin{array}{l}-0.10 \\
\quad(-0.15,-0.05)\end{array}$ & $1.83(1.32,2.34)$ & $0.24(0.13,0.38)$ & $-6.90(-6.96,-6.84)$ \\
\hline Middle-high & $\begin{array}{l}2132.59(1958.05 \\
2303.65)\end{array}$ & $\begin{array}{l}1963.64(1777.60 \\
2144.44)\end{array}$ & $\begin{array}{l}-0.35 \\
\quad(-0.38,-0.31)\end{array}$ & $0.49(0.36,0.61)$ & $0.06(0.03,0.10)$ & $-7.56(-7.76,-7.37)$ \\
\hline High & $\begin{array}{l}1248.33(1115.17 \\
1375.23)\end{array}$ & $\begin{array}{l}1238.15(1104.84 \\
1372.08)\end{array}$ & $0.02(-0.03,0.06)$ & $0.04(0.03,0.05)$ & $0.01(0.01,0.01)$ & $-6.21(-6.63,-5.80)$ \\
\hline \multicolumn{7}{|l|}{ GBD region } \\
\hline $\begin{array}{l}\text { High-income Asia } \\
\text { Pacific }\end{array}$ & $\begin{array}{l}1199.51(1067.68 \\
1331.80)\end{array}$ & $\begin{array}{l}1253.14(1096.36 \\
1415.93)\end{array}$ & $0.53(0.41,0.66)$ & $0.03(0.02,0.04)$ & $0.01(0.00,0.01)$ & $-6.22(-6.62,-5.82)$ \\
\hline Central Asia & $\begin{array}{l}2547.44(2364.30 \\
2720.15)\end{array}$ & $\begin{array}{l}2458.57(2270.29 \\
2642.65)\end{array}$ & $\begin{array}{l}-0.17 \\
\quad(-0.20,-0.15)\end{array}$ & $1.01(0.77,1.26)$ & $0.09(0.05,0.12)$ & $-8.22(-8.37,-8.08)$ \\
\hline East Asia & $\begin{array}{l}2288.85(2101.12 \\
2473.66)\end{array}$ & $\begin{array}{l}1955.43(1752.31 \\
2152.42)\end{array}$ & $\begin{array}{l}-0.47 \\
\quad(-0.53,-0.42)\end{array}$ & $1.25(0.78,1.70)$ & $0.03(0.02,0.05)$ & $\begin{array}{l}-12.76 \\
\quad(-13.47,-12.04)\end{array}$ \\
\hline South Asia & $\begin{array}{l}2262.15(2091.44 \\
2450.07)\end{array}$ & $\begin{array}{l}2396.09(2221.00, \\
2575.73)\end{array}$ & $0.19(0.17,0.21)$ & $6.53(4.99,8.18)$ & $1.67(1.10,2.44)$ & $-4.60(-4.93,-4.26)$ \\
\hline Southeast Asia & $\begin{array}{l}2064.02(1883.19 \\
2243.76)\end{array}$ & $\begin{array}{l}1980.23(1791.74 \\
2166.75)\end{array}$ & $\begin{array}{l}-0.11 \\
\quad(-0.16,-0.06)\end{array}$ & $2.46(1.23,4.77)$ & $0.36(0.14,0.66)$ & $-6.54(-6.71,-6.37)$ \\
\hline Australasia & $\begin{array}{l}1081.36(953.42 \\
1219.98)\end{array}$ & $\begin{array}{l}1172.06(1014.89 \\
1319.31)\end{array}$ & $0.28(0.13,0.44)$ & $0.01(0.00,0.01)$ & $0.01(0.00,0.01)$ & $-1.59(-2.56,-0.60)$ \\
\hline Caribbean & $\begin{array}{l}2416.16(2233.05, \\
2598.65)\end{array}$ & $\begin{array}{l}2393.01(2181.45, \\
2592.20)\end{array}$ & $\begin{array}{l}-0.06 \\
\quad(-0.08,-0.04)\end{array}$ & $0.47(0.30,0.66)$ & $0.13(0.05,0.20)$ & $-4.58(-5.03,-4.13)$ \\
\hline Central Europe & $\begin{array}{l}1888.21(1686.40 \\
2079.67)\end{array}$ & $\begin{array}{l}1724.72(1534.73, \\
1911.62)\end{array}$ & $\begin{array}{l}-0.22 \\
\quad(-0.29,-0.15)\end{array}$ & $0.08(0.05,0.12)$ & $0.00(0.00,0.00)$ & $\begin{array}{l}-10.88 \\
\quad(-11.80,-9.94)\end{array}$ \\
\hline Eastern Europe & $\begin{array}{l}1984.65(1780.53 \\
2168.39)\end{array}$ & $\begin{array}{l}2012.35(1812.10, \\
2195.27)\end{array}$ & $\begin{array}{l}-0.23 \\
\quad(-0.35,-0.11)\end{array}$ & $0.02(0.01,0.04)$ & $0.00(0.00,0.01)$ & $-6.34(-6.91,-5.76)$ \\
\hline Western Europe & $\begin{array}{l}1090.57(982.54 \\
1201.07)\end{array}$ & $\begin{array}{l}1083.39(961.16 \\
1209.27)\end{array}$ & $0.01(-0.01,0.04)$ & $0.02(0.01,0.03)$ & $0.01(0.00,0.01)$ & $-2.45(-3.09,-1.81)$ \\
\hline $\begin{array}{l}\text { Andean Latin } \\
\text { America }\end{array}$ & $\begin{array}{l}2424.94(2258.10 \\
2595.93)\end{array}$ & $\begin{array}{l}2448.14(2266.14, \\
2633.15)\end{array}$ & $0.03(0.01,0.05)$ & $0.53(0.38,0.68)$ & $0.05(0.04,0.07)$ & $-8.10(-8.77,-7.43)$ \\
\hline $\begin{array}{l}\text { Central Latin } \\
\text { America }\end{array}$ & $\begin{array}{l}2510.55(2337.69 \\
2687.12)\end{array}$ & $\begin{array}{l}2476.32(2306.59, \\
2650.84)\end{array}$ & $-0.02(-0.12,0.07)$ & $0.20(0.18,0.24)$ & $0.06(0.05,0.08)$ & $-3.61(-3.87,-3.36)$ \\
\hline $\begin{array}{l}\text { Southern Latin } \\
\text { America }\end{array}$ & $\begin{array}{l}2433.41(2190.42, \\
2643.11)\end{array}$ & $\begin{array}{l}2084.72(1847.54, \\
2315.62)\end{array}$ & $\begin{array}{l}-0.61 \\
\quad(-0.65,-0.56)\end{array}$ & $0.19(0.15,0.22)$ & $0.02(0.01,0.03)$ & $-8.65(-9.32,-7.98)$ \\
\hline $\begin{array}{l}\text { Tropical Latin } \\
\text { America }\end{array}$ & $\begin{array}{l}2177.36(2006.98 \\
2354.96)\end{array}$ & $\begin{array}{l}2004.49(1806.14, \\
2194.00)\end{array}$ & $\begin{array}{l}-0.63 \\
\quad(-0.75,-0.51)\end{array}$ & $0.16(0.12,0.22)$ & $0.03(0.02,0.04)$ & $-6.44(-6.69,-6.20)$ \\
\hline $\begin{array}{l}\text { North Africa and } \\
\text { Middle East }\end{array}$ & $\begin{array}{l}2346.16(2175.08 \\
2520.42)\end{array}$ & $\begin{array}{l}2341.72(2165.93, \\
2517.09)\end{array}$ & $\begin{array}{l}-0.06 \\
\quad(-0.09,-0.04)\end{array}$ & $2.78(0.94,4.48)$ & $0.42(0.15,0.86)$ & $-6.97(-7.41,-6.53)$ \\
\hline $\begin{array}{l}\text { High-income North } \\
\text { America }\end{array}$ & $\begin{array}{l}1134.75(1003.57 \\
1265.13)\end{array}$ & $\begin{array}{l}1180.02(1042.22 \\
1317.73)\end{array}$ & $-0.02(-0.13,0.09)$ & $0.03(0.02,0.04)$ & $0.01(0.01,0.01)$ & $-5.56(-6.42,-4.69)$ \\
\hline Oceania & $\begin{array}{l}2124.87(1928.54, \\
2323.02)\end{array}$ & $\begin{array}{l}2312.83(2096.23, \\
2514.24)\end{array}$ & $0.31(0.25,0.36)$ & $1.62(0.45,3.05)$ & $0.56(0.24,1.16)$ & $-3.28(-3.44,-3.13)$ \\
\hline $\begin{array}{l}\text { Central Sub-Saharan } \\
\text { Africa }\end{array}$ & $\begin{array}{l}2587.18(2417.58, \\
2752.66)\end{array}$ & $\begin{array}{l}2543.07(2368.10, \\
2713.33)\end{array}$ & $\begin{array}{l}-0.04 \\
\quad(-0.05,-0.03)\end{array}$ & $3.88(0.26,8.95)$ & $0.94(0.19,1.82)$ & $-4.98(-5.87,-4.09)$ \\
\hline $\begin{array}{l}\text { Eastern Sub-Saharan } \\
\text { Africa }\end{array}$ & $\begin{array}{l}2514.24(2338.12 \\
2700.57)\end{array}$ & $\begin{array}{l}2625.34(2425.49 \\
2802.26)\end{array}$ & $0.10(0.08,0.12)$ & $4.09(2.27,6.16)$ & $1.91(0.93,3.16)$ & $-2.70(-2.97,-2.42)$ \\
\hline $\begin{array}{l}\text { Southern Sub-Saha- } \\
\text { ran Africa }\end{array}$ & $\begin{array}{l}2511.09(2320.54, \\
2701.95)\end{array}$ & $\begin{array}{l}2526.16(2333.32, \\
2706.97)\end{array}$ & $0.01(0.00,0.02)$ & $0.44(0.11,0.86)$ & $0.13(0.08,0.20)$ & $-2.86(-3.22,-2.50)$ \\
\hline $\begin{array}{l}\text { Western Sub-Saharan } \\
\text { Africa }\end{array}$ & $\begin{array}{l}2546.44(2371.18, \\
2713.40)\end{array}$ & $\begin{array}{l}2552.05(2379.50, \\
2723.25)\end{array}$ & $0.00(-0.01,0.01)$ & $2.74(0.91,5.59)$ & $0.54(0.31,1.04)$ & $\begin{array}{l}-6.13(-6.53 \text { to } \\
-5.72)\end{array}$ \\
\hline
\end{tabular}

$A S I R$ age-standardized incidence rate, ASMR age-standardized mortality rate, $C I$ confidence interval, EAPC estimated annual percentage change, GBD Global Burden of Disease, SDI Socio-Demographic Index, UI uncertainty interval 

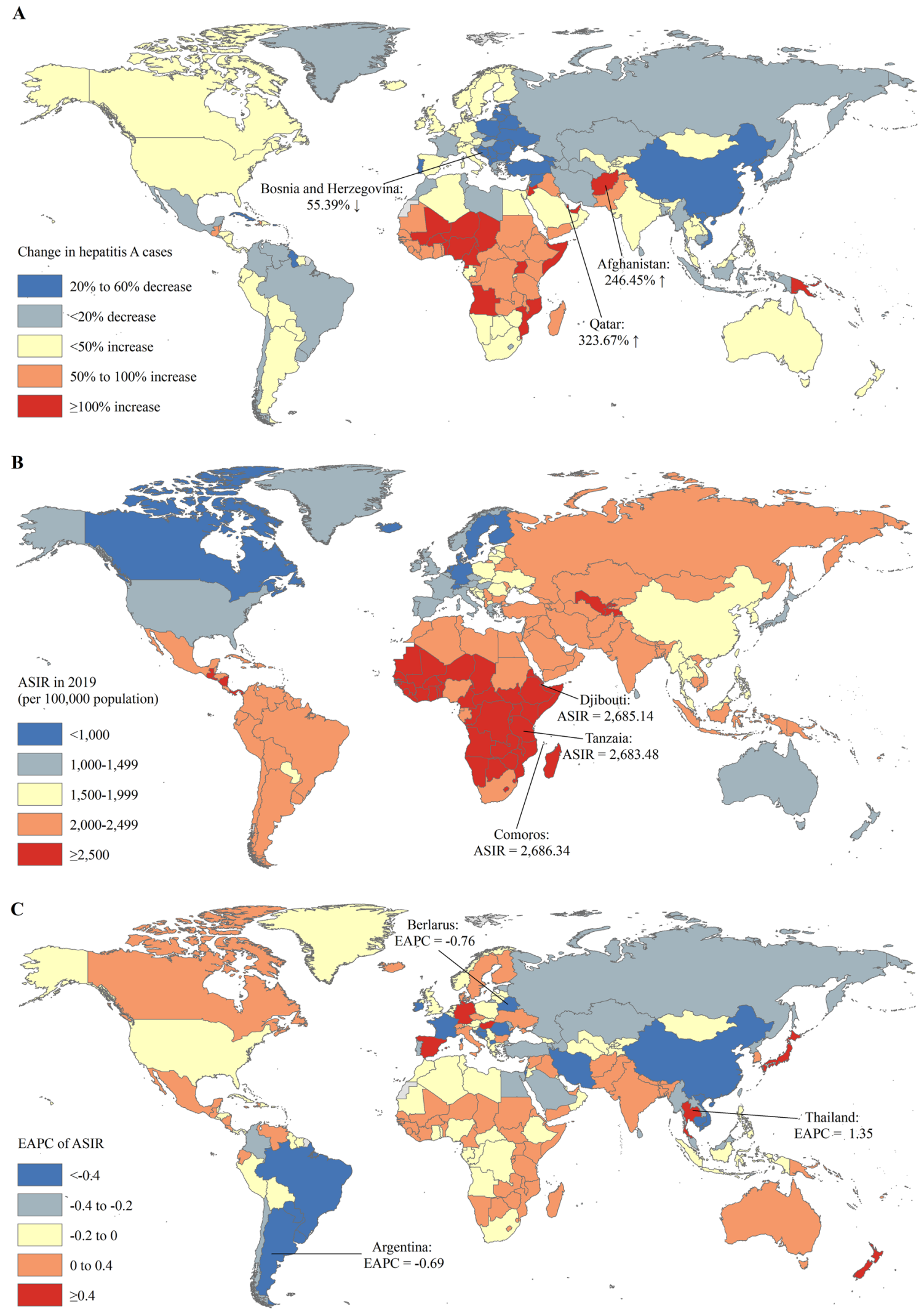

Fig. 1 The global trends in incidence of hepatitis A in 204 countries and territories. A The ASIRs of hepatitis A in 2019; B the relative change in incident cases of hepatitis A between 1990 and 2019; $\mathbf{C}$ the
EAPCs of hepatitis A ASIRs from 1990 to 2019. ASIR age-standardized incidence rate, $E A P C$ estimated annual percentage change 


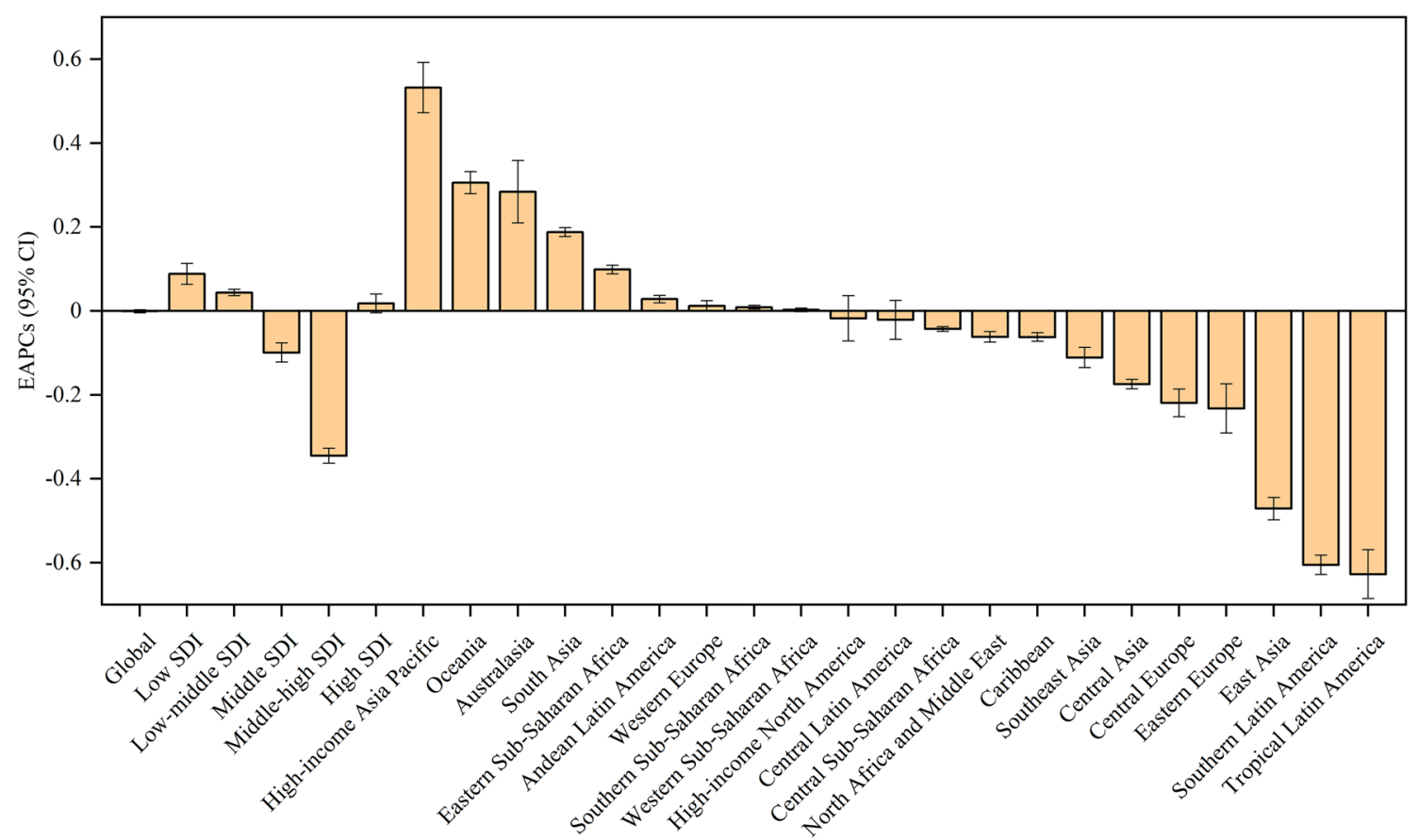

Fig. 2 The EAPCs of hepatitis A ASIRs from 1990 to 2017 at regional level. ASIR age-standardized incidence rate, EAPC estimated annual percentage change, $S D I$ socio-demographic index
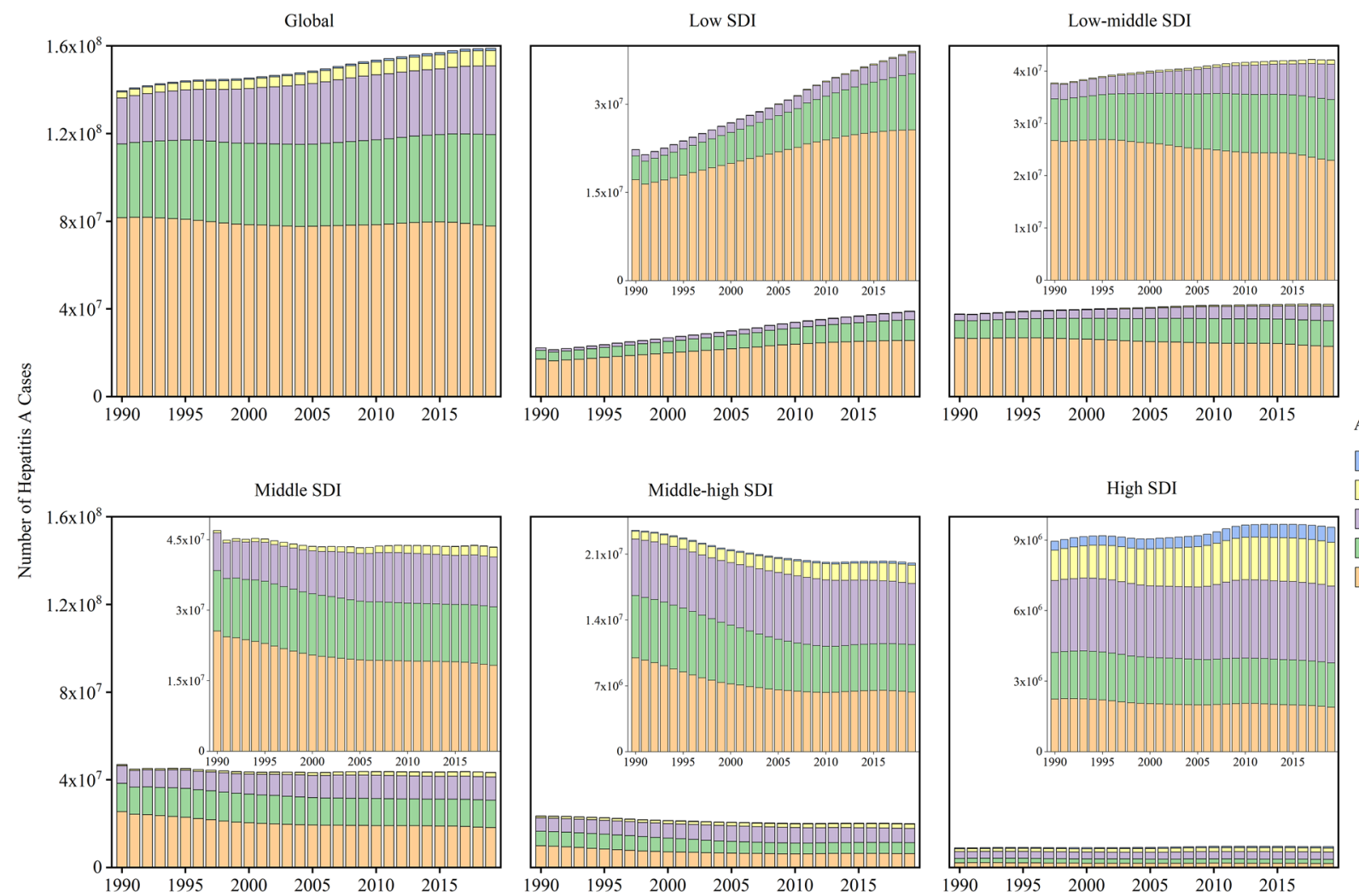

Age (years)
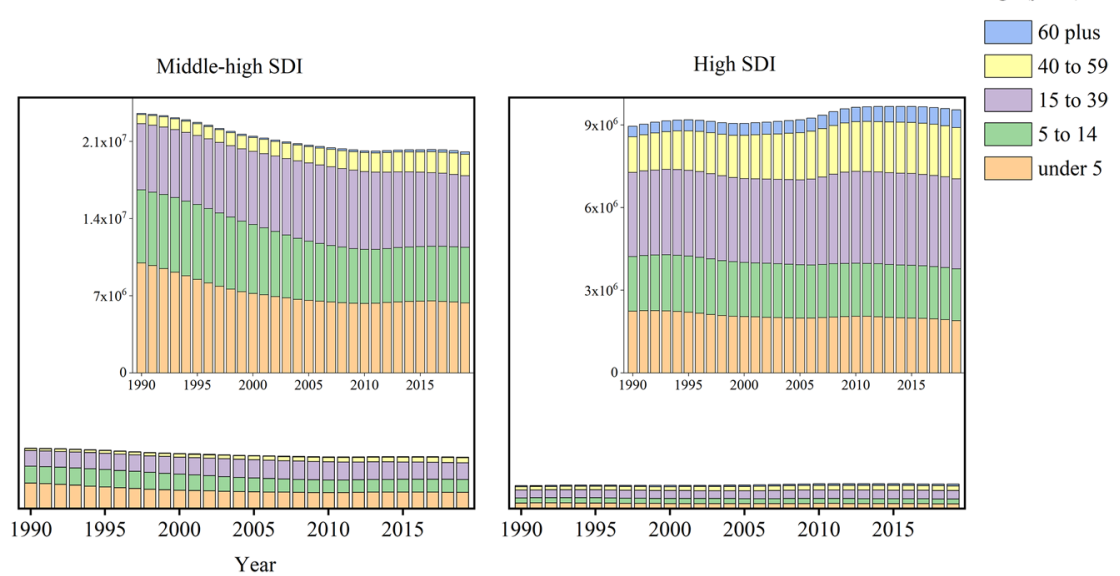

Fig. 3 The number of incident cases of hepatitis A by age group, by SDI regions, from 1990 to 2019. The data of five SDI regions are presented in the top-right panel. SDI socio-demographic index 
across the 21 GBD regions, with the highest increasing trend in high-income Asia Pacific (EAPC $=0.53 ; 95 \% \mathrm{CI}$ $0.41-0.66)$, Oceania (EAPC $=0.31 ; 95 \%$ CI $0.25-0.36$ ), and Australasia $(\mathrm{EAPC}=0.28 ; 95 \%$ CI $0.13-0.44)$; and stable in Western Europe, Central Latin America, North Africa and Middle East, and Western Sub-Saharan Africa. Nearly all middle-income regions had a decreased trend in ASIRs of hepatitis A, such as Central Asia, East Asia, and Southeast Asia (Table 2). The ASMR decreased in all 21 GBD regions, with the largest decrease in middleincome regions, such as East Asia (EAPC $=-12.76$; 95\% $\mathrm{CI}-13.47$ to -12.04$)$, Central Europe (EAPC $=-10.88$; 95\% CI -11.80 to -9.94$)$, and Southern Latin America $(\mathrm{EAPC}=-8.65 ; 95 \% \mathrm{CI}-9.32$ to -7.98$)$ (Table 2).

Figure 4 shows the proportions of incident cases of hepatitis A by age at 21 GBD regions in 1990 and 2019. The children aged under 5 years accounted for approximately $50 \%$ incident cases globally, and even about $70 \%$ in some low-income regions, including Eastern Sub-Saharan Africa and Southern Sub-Saharan Africa. All high-income regions, including high-income Asia Pacific, Australasia, Western Europe and high-income North America, had a much lower proportions of incident cases among children aged under 5 which was less than $20 \%$. However, adults aged 15-39 years had the highest proportions of incident cases in these high-income regions. In addition, the proportions of incident cases were high in older adults aged 60 years and older in these regions, even $10.18 \%$ in Western European.

\section{Factors associated with EAPC of ASIR}

A significant negative correlation was observed between EAPC and ASIR in $1990(\rho=-0.176, p=0.012)$ (Fig. 5A). Surprisingly, a significant positive correlation was detected between EAPC and SDI $(\rho=0.371, p<0.001)$ for the countries and territories with SDI value $\geq 0.7$, while a significant negative correlation was observed in the countries and territories with SDI value $<0.7(\rho=-0.310, p<0.001)$ (Fig. 5B). For SDI value $\geq 0.7$, countries or territories with higher SDI have experienced an increased trend in ASIR from 1990 to 2019.

\section{Discussion}

This study assessed the global landscape, long-term trends, and regional differences in incidence and mortality of hepatitis A using a post hoc analysis of data from the GBD study 2019. Globally, the ASIR of hepatitis A remained stable from 1990 to 2019, while the number of incident cases
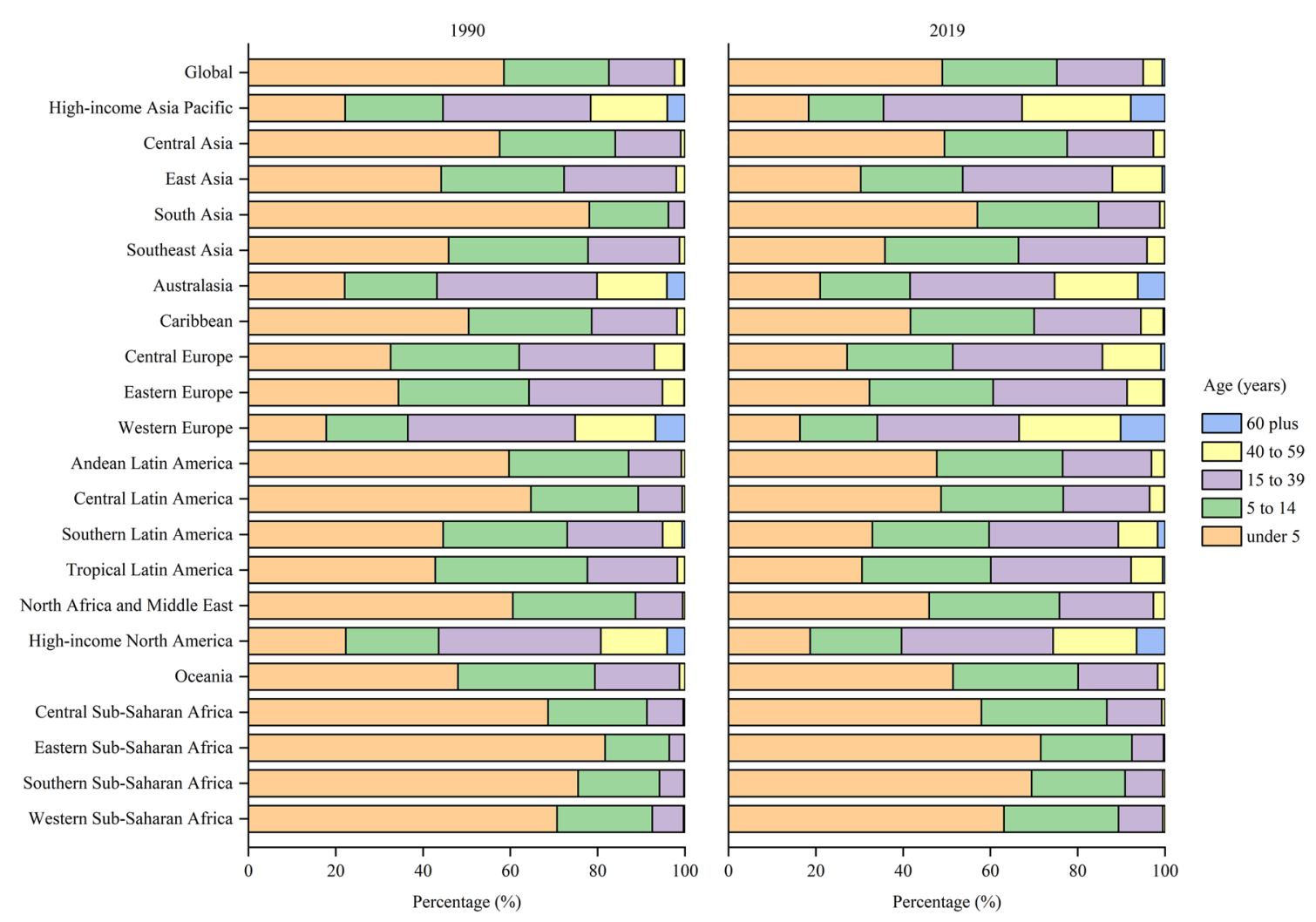

Fig. 4 The age distribution of incident cases of hepatitis A by GBD region in 1990 and 2019. GBD Global Burden of Disease 

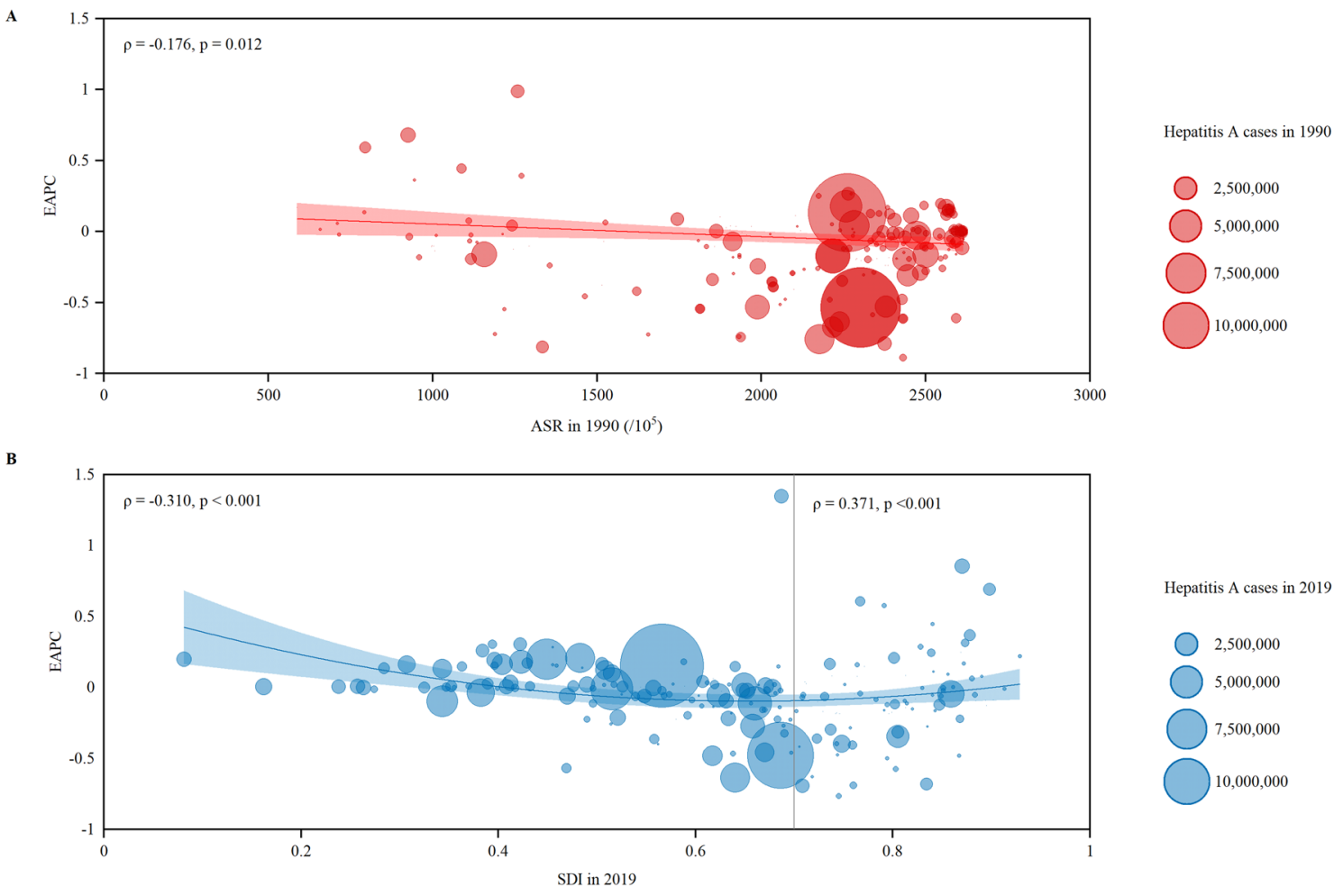

Fig. 5 The EAPCs of hepatitis A ASIRs at countries and territorial level. A The correlation between EAPC and ASIR of hepatitis A in 1990. B The correlation between EAPC and SDI in 2019. The incident cases of hepatitis A from 204 countries and territories were represented by the circles. The size of circles increased with the incident cases of hepatitis A. The $\rho$ indices and $p$ values were derived from Pearson correlation analysis. ASIR age-standardized incidence rate, $E A P C$ estimated annual percentage change, SDI socio-demographic index

A incident cases of the global in 2019 [33]. Moreover, immigrant growth may also play an important role in driving the increase of incident cases of hepatitis A. For example, we found the largest increase of incident cases of hepatitis A in Qatar where large numbers of immigrant workers often from low-income countries have been drawn in the last 2 decades [34]. In the growing population of immigrant families, pediatric travelers could be silent reservoirs of hepatitis $A$ after return from the visiting of friends and relatives travel [35]. Finally, the number of hepatitis A cases could increase with the globalization processes and increased high-risk populations of hepatitis A [24, 28].

Consistent with previous studies [29, 36], this study found the incidence of hepatitis A being closely related to socioeconomic development. We found that hepatitis A tended to be more prevalent in low-income regions, including Sub-Saharan Africa and South Asia, where HAV is highly endemic and most persons become infected in early childhood [29]. Poor sanitary and hygienic conditions contributed to the propagation of HAV in low-income regions, including household crowding, poor levels of sanitation and inadequate water supplies [29, 37]. In addition, we found a decreased trend in incidence of hepatitis $\mathrm{A}$ in most middle-income earth's inhabitants, had approximately one third of hepatitis 
regions, but an increased trend in Andean Latin America and Oceania. In middle-income regions, despite sanitary and hygienic conditions varying, nearly all populations have access to clean water and some children during their early childhood can avoid HAV infection, especially in urban areas [24, 29]. In this study, we found a lower proportions of incident cases in children aged under 5 years in middleincome regions than low-income regions. Finally, we found that the trends in incidence of hepatitis A increased in two high-income regions, including high-income Asia Pacific and Australasia. Good sanitation and hygienic conditions are available and infection rates of hepatitis $\mathrm{A}$ in children are generally low in most high-income countries [29, 37]. However, most adults are still susceptible to HAV infection and foodborne outbreaks of hepatitis A are becoming more frequent in high-income regions [24]. The peak rates of HAV infection tend to occur in adolescents and young adults. In addition, the burden of hepatitis A still significantly attribute to large community-wide outbreaks with person-to-person transmission. Finally, HAV infection predominates among persons at high risk in these high-income regions, such as travelers to countries with high endemicity levels of hepatitis A, MSM, PWIDs, and homeless persons [28, 29, 37, 38].

We found that the trends in incidence of hepatitis A decreased in most countries and territories, such as Belarus, China, and Brazil. In these countries, rising socioeconomic levels and increased access to good sanitation and hygiene facilities and clean drinking water contributed to the decreased incidence of hepatitis A [24, 36, 39]. In addition, the availability of a hepatitis A vaccine that was developed in the 1990s also played an important role in reducing this disease incidence [17, 37, 40]. For example, a shellfish-associated hepatitis A outbreak caused 300,000 cases in Shanghai, China, in two months of the first quarter of 1988 [41]. With the very rapid socioeconomic development, improved sanitary and hygienic conditions, and the introduction of hepatitis A vaccine in China, the incidence rate of hepatitis A dropped dramatically in the following couple of decades [42-44]. In addition, the decreased incidence of hepatitis A in China is also driving a decreased trend in ASIRs of hepatitis A in East Asia since China is the most populous country in East Asia. Despite the above effective ways combating hepatitis A being available for several decades, the incidence of hepatitis A still increased in high endemic countries and even some low endemic countries, such as Japan, Germany, Canada, and Italy. In countries with high endemicity levels of hepatitis A, HAV is wide spread due to poor sanitary conditions and hygienic practices, leading an increased trend in incidence of hepatitis A [24]. In the countries where the risk of HAV infection from food or water is low, there were hepatitis A outbreaks among population at high risk of hepatitis A [23, 45-50]. For example, hepatitis A outbreaks were particularly driven by transmission between non-immune MSM engaging in high-risk sexual behavior, which has been reported in European and United States populations [23, 46-49]. In addition, hepatitis A outbreaks often occurred among PWIDs, and homelessness in United States [23, 50]. Finally, globalization have driven increased international trade, migration and travel which are expected to increase foodborne outbreaks of hepatitis A in high-income countries [7, 24]. In the last decades, imported frozen berries have caused numerous foodborne outbreaks of hepatitis A in Australia and Europe [25, 51, 52]. Hepatitis A outbreaks usually occurred when residents in low endemic countries migrated and traveled to areas with high endemicity levels of HAV [24]. Evidence has reported that the hepatitis A outbreaks in Hispanic children in urban areas of the United States-Mexico border were significantly associated with cross-border travel from the United States to Mexico and foodborne exposures during the travel [53]. Therefore, facing with the complex situation of hepatitis A, more efforts should be made to eliminate hepatitis A infection globally.

In this study, a faster decreasing temporal trend in ASIR of hepatitis A between 1990 and 2019 was observed in the country with a higher baseline ASIR in 1990. It was probably because the country with higher baseline ASIR was easier to reduce the massive hepatitis A outbreaks by the development of countries' economies and public health infrastructure, as well as the introduce of hepatitis A vaccine [24]. Furthermore, we found that in the countries or territories with SDI value $\geq 0.7$, those with higher SDI have experienced an increased trend in ASIR of hepatitis A from 1990 to 2019. The possible explanations might be that the increasing age at midpoint of population immunity could increase the hepatitis A cases in high-risk populations which is more likely in high SDI countries [7]. Thus, population aging in high SDI countries could increase adults susceptible to HAV infection, leading an increased trend in hepatitis A cases and ASIR of hepatitis A in these countries [54]. In addition, outbreaks of hepatitis A among MSM have been reported in most high SDI countries in recent years, which could play an important role in increased trend in ASIR of hepatitis A in these countries [27, 55].

Although the globalization processes and increased high-risk populations of hepatitis A possibly derived an increased trend in ASIR of hepatitis A in several regions, such as high-income Asia Pacific, Oceania and Australasia, all regions have shown varying degrees of a decreased trend in ASMR of hepatitis A in this study. The decreased trend in ASMR of hepatitis A mainly benefits from vaccination strategy, increases in the quality of health care, and greater availability of liver transplantation [17, 37, 56, 57]. The globally uneven distribution of these factors mentioned above may explain the discrepancy of decreased trend in ASMR of hepatitis A across regions [58-60]. There is no specific treatment for HAV infection and treatment relies 
on supportive management, underscoring the importance of prevention. Hepatitis A vaccine can effectively prevent HAV infection in people at high mortality risk of hepatitis A, such as older adults and patients with chronic liver diseases $[17,37]$. HAV infection causes usually self-limiting hepatitis but rarely fulminant liver failure ( $<1 \%$ of cases). However, fulminant liver failure from hepatitis A may rapidly progress within a week, often resulting in death or an emergent liver transplant [61, 62]. Thus, early diagnose of hepatitis A-associated fulminant liver failure and urgent decision on liver transplantation are required [63-65]. Previous studies have reported that patients with hepatitis A-associated fulminant liver failure showed that $55-57 \%$ of patients spontaneously recovered, 31-38\% underwent liver transplantation, and $6-14 \%$ died without transplantation $[64,65]$. Survival rates of $65 \%$ or greater may be achieved by early referral for liver transplantation in patients with cerebral edema and multiple organ failure [3].

Since 1999, several countries have introduced universal HAV vaccination of children against HAV obtained high HAV vaccination coverage across these countries [59]. For instance, despite slow uptake in the first three years universal HAV vaccination program introduction in Israel, HAV vaccination coverage reached about $90 \%$ between 2003 and 2011 [59, 66]. In Greece, HAV vaccination coverage in 6-year-old children varied from 12.4 to $49.4 \%$ across different regions before universal HAV vaccination implementation and obtained more than $80 \%$ within 4 years postintroduction universal HAV vaccination [67]. Previous studies reported that anti-HAV antibody persistence was up to 15 years post-vaccination in $\geq 90 \%$ of vaccinated children [59, 68]. In adults, anti-HAV antibody persistence was up to 20 years post-vaccination and seropositivity rates could be $\geq 90 \%$ for up to 40 years and $\geq 85 \%$ for up to 50 years post-vaccination according to a mathematical modeling [69].

The risk of hepatitis A outbreaks can persist for extended time periods in unvaccinated individuals [24]. Thus, prevention measures that can reduce the risk for transmission of HAV should be emphasized. Sanitation measures play an important role in HAV infection prevention, including but not limited to careful attention to hygiene, particularly in the food service industry [35]. Heating foods to $185^{\circ} \mathrm{F}\left(85^{\circ} \mathrm{C}\right)$ for $1 \mathrm{~min}$, use of a 1:100 solution of household bleach, handwashing, and avoiding contact with uncooked foods, are all techniques that may reasonably decrease the likelihood of hepatitis A transmission [70]. In addition, prophylaxis of vulnerable populations and children through active or passive immunization is the most important prevention practice $[17,71,72]$. WHO recommends that hepatitis A vaccine be integrated into the national immunization schedule for children aged 1 year and older in countries where clinical hepatitis A is an important health problem and immunization is likely to be a cost-effective public health tool to control this disease [73]. The recommendations for vaccination varied by the countries' endemicity of hepatitis A. In high endemic countries, large-scale immunization efforts against hepatitis A should not be undertaken due to near universal immunity from asymptomatic childhood infections. Nationwide vaccination might be considered in intermediate endemic countries where the transmission of HAV occurs primarily from person to person in the general community. In low endemic countries, vaccination is considered for highrisk groups, such as MSM, PWIDs, homelessness and persons traveling to high-risk areas. The comprehensive plan for prevention and control of viral hepatitis should take surveillance and vaccination against hepatitis A into its part.

Despite vaccines against HAV can prevent hepatitis A, they are futile for hepatitis A-associated fulminant hepatitis [74-76]. In addition, hepatitis A patients had increased risk of comorbidities such as liver disease, disorders of lipid metabolism and chronic kidney disease, leading to prolonged hospitalization [75]. Therefore, anti-HAV drug and therapeutic intervention strategies for preventing progression to a life-threatening liver disease from hepatitis A are still in need. Previous studies have reported that small interfering RNAs can silence translation and replication of the firefly luciferaseencoding HAV replicon and is a most promising candidate for future therapeutic application to cure severe or fulminant cases of hepatitis A [74, 77]. Moreover, evidence suggests that HAV internal ribosomal entry-site (IRES) is an attractive target of antiviral agents against HAV and the JAK2 inhibitor AZD1480 can inhibit IRES activity and HAV replication [75, 76]. Recently, Japanese miso extracts have been reported to have inhibitory effects on HAV replication not only in patients infected with HAV but also in patients superinfected with HAV and HBV [9].

The GBD study provides a better understanding of the trend in incidence of hepatitis A over the last couple of decades globally. Some limitations in this study should also be acknowledged. First, the accuracy and robustness of GBD estimates largely depend on the quality and quantity of data used in the modeling [32]. Second, due to the GBD study taking the country as its basic unit, the incidence of hepatitis A might be a margin of bias in countries in lack of national systematic surveillance and population-based studies of hepatitis A. Third, although the standardization makes the incidence and mortality rates of hepatitis A comparable at the global, regional and national levels, the ASIR and ASMR of hepatitis A only reflect the burden of hepatitis A for one region or country under the age structure of a GBD World Standard Population.

\section{Conclusion}

In summary, there is an unfavorable trend in ASIR of hepatitis A that it is still pending in hyperendemic regions and is emerging in low endemic regions globally. Due to the rapid 
globalization processes and increased high-risk populations of hepatitis A, the number of hepatitis A cases is probably in an increasing trend, posing novel challenges for eliminating this disease, especially in high-income regions. Thus, more targeted and specific strategies were needed to eliminate hepatitis A, such as sanitation measures and a comprehensive plan for surveillance and vaccination against hepatitis A. Future studies focusing on targeted prevention and control strategies for hepatitis A were warranted.

Supplementary Information The online version contains supplementary material available at https://doi.org/10.1007/s12072-021-10232-4.

Acknowledgements We appreciate the works by the Global Burden of Disease study 2019 collaborators.

Author contributions GC searched the literature, designed the study, analyzed the data, interpreted the results, and drafted the manuscript; WJ designed the study, interpreted the results, and drafted the manuscript. JL and ML conceived the study, designed the study, supervised the study, interpreted the results, and revised the manuscript. All the authors approved the final version of the manuscript.

Funding This work was supported by the National Natural Science Foundation of China (Grant number: 71934002, 71874003) and National Key Research and Development Program of China (grant number: 2020YFC0846300). The funders had no role in study design, data collection and analysis, decision to publish, or preparation of the paper. No payment was received by any of the co-authors for the preparation of this article.

Data availability All data were obtained from the Global Health Data Exchange (GHDx) query tool (http://ghdx.healthdata.org/gbd-resul ts-tool) and the United Nations, Department of Economic and Social Affairs, Population Division (2019) (https://population.un.org/wpp/).

\section{Declarations}

Conflict of interest Guiying Cao, Wenzhan Jing, Jue Liu, Min Liu declare that they have no conflict of interest.

Animal research Not applicable.

Plant reproducibility Not applicable.

Clinical trials registration Not applicable.

Consent to participate Not applicable.

Consent to publish Not applicable.

Gels and blots Not applicable.

Open Access This article is licensed under a Creative Commons Attribution 4.0 International License, which permits use, sharing, adaptation, distribution and reproduction in any medium or format, as long as you give appropriate credit to the original author(s) and the source, provide a link to the Creative Commons licence, and indicate if changes were made. The images or other third party material in this article are included in the article's Creative Commons licence, unless indicated otherwise in a credit line to the material. If material is not included in the article's Creative Commons licence and your intended use is not permitted by statutory regulation or exceeds the permitted use, you will need to obtain permission directly from the copyright holder. To view a copy of this licence, visit http://creativecommons.org/licenses/by/4.0/.

\section{References}

1. Linder KA, Malani PN. Hepatitis A. JAMA. 2017;318(23):2393. https://doi.org/10.1001/jama.2017.17244.

2. Feinstone SM, Kapikian AZ, Purceli RH. Hepatitis A: detection by immune electron microscopy of a viruslike antigen associated with acute illness. Science. 1973;182(4116):1026-8. https://doi.org/10.1126/science.182.4116.1026.

3. Koff RS, Hepatitis A. Lancet. 1998;351(9116):1643-9. https:// doi.org/10.1016/s0140-6736(98)01304-x.

4. Wenzel JJ, Allerberger F. Hepatitis A as a foodborne infection. Lancet Infect Dis. 2014;14(10):907-8. https://doi.org/10.1016/ s1473-3099(14)70897-7.

5. WHO. WHO position paper on hepatitis A vaccines. Weekly Epidemiol Record. 2012;87(28-29):261-76 (PMID: 22905367).

6. Shin EC, Jeong SH. Natural history, clinical manifestations, and pathogenesis of hepatitis A. Cold Spring Harb Perspect Med. 2018;8(9):a031708. https://doi.org/10.1101/cshperspect.a031708.

7. Lemon SM, Ott JJ, Van Damme P, Shouval D. Type A viral hepatitis: a summary and update on the molecular virology, epidemiology, pathogenesis and prevention. J Hepatol. 2018;68:167. https:// doi.org/10.1016/j.jhep.2017.08.034.

8. Sedhom D, D'Souza M, John E, Rustgi V. Viral hepatitis and acute liver failure: still a problem. Clin Liver Dis. 2018;22(2):289-300. https://doi.org/10.1016/j.cld.2018.01.005.

9. Win NN, Kanda T, Ogawa M, et al. Superinfection of hepatitis A virus in hepatocytes infected with hepatitis B virus. Int J Med Sci. 2019;16(10):1366-70. https://doi.org/10.7150/ijms.32795.

10. Das AA-O, Barrientos R, Shiota TA-O, et al. Gangliosides are essential endosomal receptors for quasi-enveloped and naked hepatitis A virus. Nat Microbiol. 2020;5(9):1069-78.

11. Wang M, Feng ZA-O. Mechanisms of Hepatocellular Injury in Hepatitis A. Viruses. 2021;13(5):861. https://doi.org/10.3390/ v13050861.

12. Vallbracht A, Gabriel P, Maier K, Hartmann F, Steinhardt HJ, Müller C, et al. Cell-mediated cytotoxicity in hepatitis A virus infection. Hepatology. 1986;6(6):1308-14. https://doi.org/10. 1002/hep.1840060614.

13. Kim J, Chang DY, Lee HW, et al. Innate-like cytotoxic function of bystander-activated CD8(+) T cells is associated with liver injury in acute hepatitis A. Immunity. 2018;48(1):161-173.e5. https:// doi.org/10.1016/j.immuni.2017.11.025 (Epub 2018 Jan 2).

14. Hirai-Yuki A, Hensley L, McGivern DR, et al. MAVS-dependent host species range and pathogenicity of human hepatitis A virus. Science. 2016;353(6307):1541-5. https://doi.org/10.1126/scien ce.aaf8325.

15. Belkaya SA-OX, Michailidis EA-O, Korol CB, et al. Inherited IL$18 \mathrm{BP}$ deficiency in human fulminant viral hepatitis. J Exp Med. 2019;216(8):1777-90. https://doi.org/10.1084/jem.20190669.

16. Nainan OV, Xia G, Vaughan G, Margolis HS. Diagnosis of hepatitis a virus infection: a molecular approach. Clin Microbiol Rev. 2006;19(1):63-79. https://doi.org/10.1128/CMR.19.1.63-79.2006.

17. Zhang L. Hepatitis A vaccination. Hum Vaccin Immunother. 2020;16(7):1565-73. https://doi.org/10.1080/21645515.2020. 1769389.

18. Lifely MR, Rogers MV, Esdaile J, Payne M, Tite JP. Murine crossreactive T-cell epitopes of Neisseria meningitidis outer membrane 
proteins. Vaccine. 1992;10(3):159-63. https://doi.org/10.1016/ 0264-410x(92)90005-5.

19. Werzberger A, Mensch B, Kuter B, et al. A controlled trial of a formalin-inactivated hepatitis A vaccine in healthy children. $\mathrm{N}$ Engl J Med. 1992;327(7):453-7. https://doi.org/10.1056/nejm1 99208133270702.

20. Xu Z, Wang X, Li R, et al. Immunogenicity and efficacy of two live attenuated hepatitis A vaccines $(\mathrm{H}(2)$ strains and LA-1 strains). Zhonghua Yi Xue Za Zhi. 2002;82(10):678-81 (PMID: 12133465)

21. Mao JS, Chai SA, Xie RY, et al. Further evaluation of the safety and protective efficacy of live attenuated hepatitis A vaccine (H2-strain) in humans. Vaccine. 1997;15(9):944-7. https://doi. org/10.1016/s0264-410x(96)00304-0.

22. Hu X, Collier MG, Xu F. Hepatitis A outbreaks in developed countries: detection, control, and prevention. Foodborne Pathog Dis. 2020;17(3):166-71. https://doi.org/10.1089/fpd.2019.2648.

23. Foster MA, Hofmeister MG, Kupronis BA, et al. Increase in hepatitis A virus infections-United States, 2013-2018. MMWR Morb Mortal Wkly Rep. 2019;68(18):413-5. https://doi.org/10.15585/ mmwr.mm6818a2.

24. Jacobsen KH. Globalization and the Changing Epidemiology of Hepatitis A Virus. Cold Spring Harb Perspect Med. 2018;8(10):a031716. https://doi.org/10.1101/cshperspect.a0317 16.

25. Bozkurt H, Phan-Thien KY, van Ogtrop F, Bell T, McConchie R. Outbreaks, occurrence, and control of norovirus and hepatitis a virus contamination in berries: a review. Crit Rev Food Sci Nutr. 2021;61(1):116-38. https://doi.org/10.1080/10408398.2020. 1719383.

26. Greig JD, Ravel A. Analysis of foodborne outbreak data reported internationally for source attribution. Int J Food Microbiol. 2009;130(2):77-87. https://doi.org/10.1016/j.ijfoodmicro.2008. 12.031 .

27. Urbanus AT, Van Houdt R, van de Laar TJ, Coutinho RA. Viral hepatitis among men who have sex with men, epidemiology and public health consequences. Euro Surveill. 2009;14(47):19421. https://doi.org/10.2807/ese.14.47.19421-en.21.

28. Franco E, Giambi C, Ialacci R, Coppola RC, Zanetti AR. Risk groups for hepatitis A virus infection. Vaccine. 2003;21(1920):2224-33. https://doi.org/10.1016/s0264-410x(03)00137-3.

29. Jacobsen KH, Wiersma ST. Hepatitis A virus seroprevalence by age and world region, 1990 and 2005. Vaccine. 2010;28(41):66537. https://doi.org/10.1016/j.vaccine.2010.08.037.

30. Global Burden of Disease Collaborative Network (2020) Global Burden of Disease Study 2019 (GBD 2019) Results. Seattle, United States: Institute for Health Metrics and Evaluation (IHME), 2020. Available from http://ghdx.healthdata.org/gbdresults-tool.

31. GBD 2019 Diseases and Injuries Collaborators. Global burden of 369 diseases and injuries in 204 countries and territories, 1990-2019: a systematic analysis for the Global Burden of Disease Study 2019. Lancet 2020;396(10258):1204-1222. https://doi. org/10.1016/s0140-6736(20)30925-9

32. Liu Z, Jiang Y, Yuan H, et al. The trends in incidence of primary liver cancer caused by specific etiologies: Results from the Global Burden of Disease Study 2016 and implications for liver cancer prevention. J Hepatol. 2019;70(4):674-83. https://doi.org/10. 1016/j.jhep.2018.12.001.

33. United Nations, Department of Economic and Social Affairs, Population Division (2019). World Population Prospects 2019, Online Edition. Rev. 1. https://population.un.org/wpp/.

34. Abu-Madi MA, Behnke JM, Ismail A, Al-Olaqi N, Al-Zaher $\mathrm{K}$, El-Ibrahim R. Comparison of intestinal parasitic infection in newly arrived and resident workers in Qatar. Parasit Vectors. 2011;4:211. https://doi.org/10.1186/1756-3305-4-211.

35. Trujillo-Ochoa JL, Viera-Segura O, Fierro NA. Challenges in management of hepatitis A virus epidemiological transition in Mexico. Ann Hepatol. 2019;18(1):14-22. https://doi.org/10.5604/ 01.3001.0012.7857.

36. Jacobsen KH, Koopman JS. The effects of socioeconomic development on worldwide hepatitis A virus seroprevalence patterns. Int J Epidemiol. 2005;34(3):600-9. https://doi.org/10.1093/ije/ dyi062.

37. Wasley A, Fiore A, Bell BP. Hepatitis A in the era of vaccination. Epidemiol Rev. 2006;28:101-11. https://doi.org/10.1093/epirev/ mxj012.

38. Sane J, MacDonald E, Vold L, Gossner C, Severi E. Multistate foodborne hepatitis A outbreak among European tourists returning from Egypt-need for reinforced vaccination recommendations, November 2012 to April 2013. Euro Surveill. 2015. https://doi. org/10.2807/1560-7917.es2015.20.4.21018.

39. Franco E, Meleleo C, Serino L, Sorbara D, Zaratti L. Hepatitis A: Epidemiology and prevention in developing countries. World J Hepatol. 2012;4(3):68-73. https://doi.org/10.4254/wjh.v4.i3.68.

40. WHO position paper on hepatitis (2012) A vaccines-June 2012. Wkly Epidemiol Rec 87(28/29):261-276. PMID: 22905367.

41. Halliday ML, Kang LY, Zhou TK, et al. An epidemic of hepatitis A attributable to the ingestion of raw clams in Shanghai. China J Infect Dis. 1991;164(5):852-9. https://doi.org/10.1093/infdis/ 164.5.852.

42. Sun XJ, Zhang GM, Zhou RJ, et al. Changes in the epidemiology of hepatitis A in three socio-economic regions of China, 19902017. Infect Dis Poverty. 2019;8(1):80. https://doi.org/10.1186/ s40249-019-0591-z.

43. Wang F, Sun X, Wang F, et al. Changing Epidemiology of Hepatitis A in China: evidence from three National Serological Surveys and the National Notifiable Disease Reporting System. Hepatology. 2021;73(4):1251-60. https://doi.org/10.1002/hep.31429.

44. Cui F, Liang X, Wang F, Zheng H, Hutin YJ, Yang W. Development, production, and postmarketing surveillance of hepatitis A vaccines in China. J Epidemiol. 2014;24(3):169-77. https://doi. org/10.2188/jea.je20130022.

45. Sachdeva H, Benusic M, Ota S, et al. Community outbreak of hepatitis A disproportionately affecting men who have sex with men in Toronto, Canada, January 2017-November 2018. Can Commun Dis Rep. 2019;45(10):262-8. https://doi.org/10.14745/ ccdr.v45i10a03.

46. Ndumbi P, Freidl GS, Williams CJ, et al. Hepatitis A outbreak disproportionately affecting men who have sex with men (MSM) in the European Union and European Economic Area, June 2016 to May 2017. Euro Surveill. 2018. https://doi.org/10.2807/15607917.es.2018.23.33.1700641.

47. Bordi L, Rozera G, Scognamiglio P, et al. Monophyletic outbreak of Hepatitis A involving HIV-infected men who have sex with men, Rome, Italy 2008-2009. J Clin Virol. 2012;54(1):26-9. https://doi.org/10.1016/j.jcv.2012.01.009.

48. Mazick A, Howitz M, Rex S, et al. Hepatitis A outbreak among MSM linked to casual sex and gay saunas in Copenhagen. Denmark Euro Surveill. 2005;10(5):111-4 (PMID: 16077208).

49. Taffon $\mathrm{S}$, Bidini $\mathrm{G}$, Vichi $\mathrm{F}$, et al. A unique HAV strain circulated in patients with acute HAV infection with different risk exposures in Tuscany. Italy J Clin Virol. 2011;50(2):142-7. https://doi.org/ 10.1016/j.jcv.2010.10.011.

50. Nelson R. Hepatitis A outbreak in the USA. Lancet Infect Dis. 2018;18(1):33-4. https://doi.org/10.1016/s1473-3099(17) 30718-1.

51. Tavoschi L, Severi E, Niskanen T, et al. Food-borne diseases associated with frozen berries consumption: a historical perspective, 
European Union, 1983 to 2013. Euro Surveill. 2015;20(29):21193. https://doi.org/10.2807/1560-7917.es2015.20.29.21193.

52. Franklin N, Camphor H, Wright R, Stafford R, Glasgow K, Sheppeard V. Outbreak of hepatitis A genotype IB in Australia associated with imported frozen pomegranate arils. Epidemiol Infect. 2019;147: e74. https://doi.org/10.1017/s0950268818003515.

53. Weinberg M, Hopkins J, Farrington L, Gresham L, Ginsberg M, Bell BP. Hepatitis A in Hispanic children who live along the United States-Mexico border: the role of international travel and food-borne exposures. Pediatrics. 2004;114(1):e68-73. https://doi. org/10.1542/peds.114.1.e68.

54. Carrion AF, Martin P. Viral hepatitis in the elderly. Am J Gastroenterol. 2012;107(5):691-7. https://doi.org/10.1038/ajg.2012.7.

55. Stene-Johansen K, Tjon G, Fau - Schreier E, Schreier E Fau Bremer V, , et al. Molecular epidemiological studies show that hepatitis A virus is endemic among active homosexual men in Europe. J Med Virol. 2007;79(4):356-65. https://doi.org/10.1002/ jmv.20781.

56. Vitral CL, Gaspar Am Fau - Souto FJD, Souto FJ (2006) Epidemiological pattern and mortality rates for hepatitis A in Brazil, 1980-2002 - a review. Mem Inst Oswaldo Cruz 101(2):119-127. https://doi.org/10.1590/s0074-02762006000200001.

57. Jeong SH, Lee HS. Hepatitis A: clinical manifestations and management. Intervirology. 2010;53(1):15-9. https://doi.org/10.1159/ 000252779 .

58. Aggarwal R, Goel A. Hepatitis A: epidemiology in resource-poor countries. Curr Opin Infect Dis. 2015;28(5):488-96. https://doi. org/10.1097/QCO.0000000000000188.

59. Andani A, van Damme P, Bunge EM, Salgado F, van Hoorn RC, Hoet B (2021) One or two doses of hepatitis A vaccine in universal vaccination programs in children in 2020: A systematic review. Vaccine. 2021:S0264-410X(21)00054-2. doi: https://doi.org/10. 1016/j.vaccine.2021.01.038.

60. Cooke GS, Andrieux-Meyer I, Applegate TL, et al. Accelerating the elimination of viral hepatitis: a Lancet Gastroenterology \& Hepatology Commission. Lancet Gastroenterol Hepatol. 2019;4(2):135-84. https://doi.org/10.1016/S2468-1253(18) 30270-X.

61. Cuthbert JA. Hepatitis A: old and new. Clin Microbiol Rev. 2001;14(1):38-58. https://doi.org/10.1128/CMR.14.1.38-58.2001.

62. Iorio N, John S. Hepatitis A (2019) In: StatPearls [Internet]. Treasure Island (FL): StatPearls Publishing; 2021 Jan. PMID: 29083664.63.Kim JA-O, Cho EA-O, Ahn C, et al. A Model to Predict 1-Month Risk of Transplant or Death in Hepatitis A-Related Acute Liver Failure. Hepatology. 2019;70(2):621-629. https://doi. org/10.1002/hep.30262.

63. Taylor RM, Davern T, Fau - Munoz S, Munoz S Fau - Han $\mathrm{S}-\mathrm{H}$, , et al. Fulminant hepatitis A virus infection in the United States: incidence, prognosis, and outcomes. Hepatology. 2006;44(6):1589-97. https://doi.org/10.1002/hep.21439.

64. Kim JM, Fau LY, Lee -, JH, Lee Jh Fau - Kim W, Kim W Fau, Lim KS, Lim KS, . Clinical outcomes and predictive factors of spontaneous survival in patients with fulminant hepatitis A. Korean J Hepatol. 2008;14(4):474-82. https://doi.org/10.3350/kjhep.2008. 14.4.474.

65. Levine H, Kopel E Fau-Anis E, Anis E Fau - Givon-Lavi N, Givon-Lavi N Fau - Dagan R, Dagan R (2015) The impact of a national routine immunisation programme initiated in 1999 on Hepatitis A incidence in Israel, 1993 to 2012. Euro Surveill 20(7):3-10. https://doi.org/10.2807/1560-7917.
66. Mellou K, Sideroglou T, Papaevangelou V, et al. Considerations on the current universal vaccination policy against hepatitis A in Greece after recent outbreaks. PLoS ONE. 2015;10(1): e0116939. https://doi.org/10.1371/journal.pone.0116939.

67. Dagan R, Ashkenazi S Fau - Livni G, Livni G Fau - Go O, Go O Fau - Bagchi P, Bagchi P Fau - Sarnecki M, Sarnecki M (2016) Long-term Serologic Follow-up of Children Vaccinated with a Pediatric Formulation of Virosomal Hepatitis A Vaccine Administered With Routine Childhood Vaccines at 12-15 Months of Age. Pediatr Infect Dis J 35(7):e220-e2288. https://doi.org/10. 1097/INF.0000000000001176.

68. Theeten H, Van Herck K, Van Der Meeren O, Crasta P, Van Damme P, Hens N. Long-term antibody persistence after vaccination with a 2-dose Havrix (inactivated hepatitis A vaccine): 20 years of observed data, and long-term model-based predictions. Vaccine. 2015;33(42):5723-7. https://doi.org/10.1016/j.vaccine. 2015.07.008.

69. Matheny SC, Kingery JE. Hepatitis A. Am Fam Physician. 2012;86(11):1027-34 (quiz 1010-1022. PMID: 23198670).

70. Hilleman MR. Hepatitis and hepatitis A vaccine: a glimpse of history. J Hepatol. 1993;18(Suppl 2):S5-10. https://doi.org/10.1016/ s0168-8278(05)80370-8.

71. Fisenka EG, Germanovich FA, Glinskaya IN, Lyabis OI, Rasuli AM. Effectiveness of universal hepatitis A immunization of children in Minsk City, Belarus: four-year follow-up. J Viral Hepat. 2008;15(Suppl 2):57-61. https://doi.org/10.1111/j.1365-2893. 2008.01031.x.

72. WHO (2018) Hepatitis A: Vaccine Preventable Diseases Surveillance Standards. Available from: http://apps.who.int/iris/ bitstream/handle/10665/204501/9789241549547_eng.pdf?seque nce $=1$.

73. Kusov Y, Kanda T Fau - Palmenberg A, Palmenberg A Fau - Sgro J-Y, Sgro Jy Fau - Gauss-Müller V, Gauss-Müller V (2006) Silencing of hepatitis A virus infection by small interfering RNAs. J Virol 80(11):5599-5610. https://doi.org/10.1128/JVI.01773-05.

74. Jiang $X$, Kanda $T$, Nakamoto $S$, et al. The JAK2 inhibitor AZD1480 inhibits hepatitis A virus replication in Huh7 cells. Biochem Biophys Res Commun. 2015;458(4):908-12. https:// doi.org/10.1016/j.bbrc.2015.02.058

75. Jiang X, Kanda T, Wu S, et al. Suppression of La antigen exerts potential antiviral effects against hepatitis A virus. PLoS ONE. 2014;9(7): e101993. https://doi.org/10.1371/journal.pone.01019 93.

76. Kanda T, Kusov Y Fau - Yokosuka O, Yokosuka O Fau - GaussMüller V, Gauss-Müller V (2004) Interference of hepatitis A virus replication by small interfering RNAs. Biochem Biophys Res Commun 318(2):341-345. https://doi.org/10.1016/j.bbrc.2004 03.194.

77. Jing W, Liu J, Liu M. The global trends and regional differences in incidence of HEV infection from 1990 to 2017 and implications for HEV prevention. Liver Int. 2021;41(1):58-69. https://doi.org/ 10.1111/liv.14686.

Publisher's Note Springer Nature remains neutral with regard to jurisdictional claims in published maps and institutional affiliations. 